\title{
Building Communities for Change: An Experience in Mumbai
}

\author{
Varna Sri Raman Anuragini Nagar Ira Joshi \\ Sesame Workshop India Trust \\ India
}

\begin{abstract}
This paper documents Sesame Workshop India Trust's work in impoverished communities in Mumbai with regard to the provision of Early Childhood Care and Education (ECCE) services. The model works with children aged birth to six who are served by the Integrated Child Development Scheme (ICDS), sponsored by the ministry of Women and Child Development (WCD), India. The model focuses on augmenting the capacities of the anganwadi (child care center) worker vis-à-vis pedagogy and access to material, and also works with caregivers advocating for a better ECCE environment. Integrating innovative technology as well as traditional Teaching Learning Materials (TLM) and operating at a scale of five thousand plus centers, the model demonstrates how programmes can be designed from ground-up, to be inclusive, scalable and successful in improving children's learning outcomes.
\end{abstract}

Key words : Sesame Worship India Trust, early childhood care and education services, child care center, reducing poverty

\section{Introduction}

The provision of Early Childhood Care and Education (ECCE) is critical for improving performance in the first years of primary school; which feeds into the overarching goal of reducing poverty(Nations, 2009), and improving health (UNESCO, 2002). Maharashtra is the second largest state in the country and is home to its capital state Mumbai on the west coast of India.

Home to approximately twenty one million people, Mumbai, accounts for almost two percent of the

An earlier version of this paper was presented at 2011 ARNEC Conference, Singapore.

Correspondence concerning this article should be addressed to Varna Sri Raman, Sesame Worship India, E-1A, Kailash Colony, New Delhi - 110048, India. Electronic mail may be sent to varna.sr@sesameworkshopindia.org country's total population. Over forty percent of this population lives in slums. Between forty to sixty percent of Mumbai's slum children aged up to four years are malnourished, and between eight percent and thirty percent are severely malnourished (Vora, 2010). The scale of malnutrition in Mumbai poses a serious challenge to the delivery of government services, in particular, the thirty five year old Integrated Child Development Services (ICDS) Services.

Established under the Ministry of Women and Child Development, the ICDS Scheme is one of the world's largest and most unique programmes for early childhood development. The ICDS reaches out to mothers and children of socially and economically underprivileged communities and provides them with an integrated programme of health, nutrition and early childhood education. 
These services are delivered to the community ${ }^{2}$ through Anganwadi Centres (AWC) which include the following:

- Supplementary nutrition

- Immunisation

- Health checkups

- Referral services

- Non-formal pre-school education for children between three and six years of age

- Nutrition and health education

\section{Background}

The ICDS in Mumbai caters to a total population of over four lakh sixty thousand children of which almost five hundred thousand are children aged between birth and six years (ICDS Maharashtra, year). Given the severity of the problems, the focus of the ICDS remains improvement in immunization, nutrition \& health status of women \& children through service delivery. Mumbai has five thousand one hundred and fifty anganwadis ${ }^{3}$, covered by thirty three different government schemes. Poor infrastructure, vacant posts, low motivation amongst frontline workers (anganwadi workers \& supervisors) contribute to this over-all dismal situation in Mumbai (WCD, 2011). Furthermore, the Anganwadi Worker (AWW) has several responsibilities (aside of ECCE) but is inadequately trained in ECCE. This situation coupled with an increasing demand for AWC (ICDS, year) which is mostly for food served as opposed to ECCE (as a wide gamut of early childhood related services) clearly points towards an unmet need.

Given the competing demands made by the system on AWW, ECCE is often left-out when nutrition takes center stage as a major concern. The World Bank and

2 Members of the community include pregnant and lactating mothers, children and adolescent girls.

3 As on date other institutions have recommended for more active participation of community members, communication of the benefits of preschool education to parents and better training for AWW along with other improvements.

Based on the reality of ICDS in Mumbai, Sesame Workshop India Trust (SWIT) has formulated a holistic and collaborative program to strengthen the ECCE component of ICDS in all AWCs in Mumbai. The following pages describe the model adopted by SWIT including methodology and rationale while documenting lessons learnt.

\section{Objectives and Outcomes}

SWIT's mandate is to help children prepare better for school and life. As a part of its core mission-driven activities, with the Mumbai - Strengthen ECCE for ICDS programme our objective has been not replace existing government structures, but to augment and build existing capacities on the ground using innovative technologies and media in a participatory manner. The focus is improving developmentally appropriate learning outcomes in four key areas viz. Pre-literacy, Pre-numeracy, Health and Hygiene and Nutrition. Some of the key outcomes that we impact:

- Pre-literacy: Increase children's vocabulary by learning new words, the ability to narrate events, the ability to create imaginative stories and encouraging children to scribble and engage in pre-writing activities ${ }^{4}$

- Pre-numeracy: Identifying and recognizing 2D shapes, Understand and apply relational concepts (Up and down -Inside and out -Big and small -More and less -Up and down -Forward and behind) $)^{5}$

- Health and Hygiene: Familiarizing children

\footnotetext{
4 The Story Pond and Scribbles Kit

5 The Shapes Kit
} 
with the concept of a balanced meal, encouraging children to learn the value of regular meals, making healthy choices, healthy foods and their benefits to the body and identify and name healthy foods ${ }^{6}$.

Nutrition: Familiarizing children with the concept of a balanced diet, and encourage in children to learn the habit and send of decent personal hygiene habits including tooth brushing, washing hands, bathing, combing hair, cutting nails. Third-party evaluation research (BGM Policy Innovations 2010; 2011) has shown statistically significant improvements from baseline to endline in critical areas including improvements at school-readiness.

\section{Method}

Programme design at SWIT is the result of formative evaluation on content areas which suggests what key areas of focus should be for learningoutcome based improvements. The programme itself was first piloted and then scaled-up with additional components. The key processes included the following:

- Need Analysis-Research (choosing appropriate geographies for impact)

- Formative Research-Content Areas (learning outcomes)

- Pilot - Program Intervention (programme design)

- Review

- Scale - Up (Additional components)

- ME\&R and Evaluation Research - Research and Program

\section{Supporting Government Systems to Deliver ECCE Services - GGSS's Outreach Methods:}

Galli Galli Sim Sim is a multi-platform initiative that

6 The Nutrition Kit combines the power of television, radio and educational outreach to help children prepare better for school and life. One pillar of this initiative is the local co-production of the world renowned Sesame Street series. The series currently airs five times a week on Cartoon Network and POGO (Ac Neilson \& Company, 2011).

In addition to early literacy and math skills, the show also focuses on promoting good nutrition, hygiene, and healthy habits along with empathy, appreciation of differences and a sense of civic responsibility. The second pillar of the initiative is an extensive educational outreach program using three different platforms. The first platform is the Mobile Community Viewing (MCV). The $\mathrm{MCV}$ uses a repurposed vegetable cart, which is branded and houses a television and DVD player powered by batteries.

The cart rolls down 'gallis 7 ' in densely populated slum areas, to a mapped route. The mapping (routedetermination) is undertaken with a focus on reaching the maximum number of children and their caregivers.

The cart is designed to be colorful and is therefore attractive to both children and caregivers. An implementation agency, identified \& trained by SWIT interacts with the community to understand the best places and times where the cart can be stationed for the duration of the show. Once the children have gathered, a Galli Galli Sim Sim episode is played to the viewers. These episodes are chosen based on thematic requirements.

This viewing is accompanied by a variety of workshops and activities for kids (role- playing, mask making, theater etc.), which includes a distribution of materials at the end of the activity to both children and their caregivers to help ensure engagement, participation and retention of messages.

7 A Galli refers to small by-lanes, usually connecting different parts of a colony. 
The MCV aims to reach a large number of children with audio/visual content like episodes on health, hygiene, nutrition, numbers, alphabets and many others, from the television show and serves as a platform to advocate for the importance of ECCE.

The MCV aims to reach a large number of children with audio/visual content like episodes on health, hygiene, nutrition, numbers, alphabets and many others, from the television show and serves as a platform to advocate for the importance of ECCE.

The second platform is the child care centre intervention, which includes the development and distribution of print and audio/visual educational materials/kits for children and caregivers through partnerships with the government.

\section{Building Communities of Change - Impact at Scale:}

While almost all of Mumbai is served by ICDS NGOs, private schools and other funded programs also contribute to reach out to pre-schoolers in the city. Despite an increase in the establishment of AWCs, the actual outreach and coverage for early childhood education (quality \& quantity) remains unfulfilled (ICDS, year). Teaching and training quality followed by timely enrolment of children and retention in the AWCs for ECCE have been key challenges for the ICDS in Mumbai.

Each AWC is managed by an AWW, who caters to about thirty children between the ages of three to six. The ICDS therefore provides ECCE services to about one lakh sixty thousand children and their caregivers. The centres, whose mandate is, to provide necessary skills for 'whole-child development' ${ }^{\text {, }}$, have become centres where children too young to be initiated to the $3 R^{\prime} s^{9}$ are being made school-ready.

8 Development has several inter-related dimensions. These include physical, cognitive, social, spiritual and emotional development, each of which influence the other and all of which are developing simultaneously-National Curriculum Framework, India, 2005.

9 Refers to Reading, Writing and Arithmetic--Understanding
SWIT has been working with the ICDS to strengthen the quality of ECCE in Mumbai. A project was piloted with two hundred centers in November, 2009 and was scaled up in 2010 to cater to an additional four thousand three hundred AWCs. In 2011, the program now caters to over five thousand AWCs in Mumbai with new educational kits being given out each year.

The scaled up program (in 2010) included ${ }^{10}$ the following components:

1. Two kits ${ }^{11}$ (Health $\mathcal{E}$ Hygiene ${ }^{12}$ and Pre-Literacy ${ }^{13}$ ), per center which included materials for children and caregivers ${ }^{14}$.

2. Capacity development of a hundred and fifty supervisors as master trainers

3. Training of four thousand three hundred AWWs by master trainers twice in the year

4. Monitoring, Evaluation and Reporting (ME\&R) consisting of spot-checks at repeated intervals by

Our Civic Issues- Early Childhood Care and Education, The Bombay Community Public Trust.

10 Kits change every year, other components remain the same.

11 A Kit comprises of different components provided for a particular theme and can include story books, activity sheets, flash cards, coloring books etc., all put together in a GGSS-branded bag.

12 The Health \& Hygiene kit consists of a set of story books, instruction cards for the AWW, personal hygiene flash cards, large cut outs of personal hygiene items (tooth brush, soap, bucket of water, nail cutter etc), hand washing sequence cards and an accordion all put together in a GGSS branded bag.

13 The Pre-Literacy kit consists of a set of story books, instruction cards for the AWW, a floor activity sheet, portable (plastic) blackboards, chalk boxes all put together in a GGSS branded bag.

14 Print materials are designed to foster educational concepts in a fun way through the use of pictures which help increase appeal for children. Minimal use of text and instruction ensures that they are easy to implement by the AWWs in the centres and also for caregivers with no or low literacy levels. 
a monitoring agency, internal field staff and reporting at regular frequencies on progress and mid-course corrections.

5. Third-party evaluation research to investigate the impact of the intervention

6. A telephone-based support service to provide real time training, monitoring and support to supervisors, Child Development Project Officers (CDPOs) and the AWWs. ${ }^{15}$

Working in close partnership with ICDS Mumbai the program ensures that AWWs get adequate training, sufficient educational-support materials and constant supervision/support to implement ECCE in their centers. The programme also ensures that the children are provided with developmentally appropriate and fun materials to prepare them for school and life.

A good quality ECCE curriculum engages children in age appropriate activities, empowers them and provides opportunities to be an active participant in the learning process(NCERT, 2006). A curriculum which is a downward extension of primary curriculum focusing on reading, writing and arithmetic can have detrimental effects on young children who are not developmentally prepared. Implementing it in rigid-manner results in academic pressures or boredom towards education (Kaul, Sankar \& NUEPA., 2009).

15 The system consist of three main components: 1) call-outs to all supervisors and AWWs to inform them about the "activity of the week" for use with GGSS materials (2) a toll-free support line, which allows AWWs and supervisors to call in and leave a message with a question, share a success story, etc. to which the GGSS ground team responds personally; and 3) Call-outs to poll supervisors about frequency of use of materials and feedback on specific materials. This has helped training, and support in real time - to the Anganwadi centres distributed geographically and promoted the effective and frequent use of GGSS materials by teachers to help improve learning outcomes of children.

\section{The Mumbai Model - Programme Design:}

The Mumbai model programme, as on 2011, covers all five thousand one hundred and twenty eight AWCs in the Mumbai region. Partnering with the ICDS in Mumbai presents SWIT with the opportunity to impact the lives of community members and young children through a comprehensive outreach programme.

The programme design focused on:

- Support for Early Learning At Home

- AWW Capacity Building

- Joyful Learning Experiences

- Early Learning at Home

Learning for a child of any age begins at home. It is therefore critical to create home-environments which are supportive of learning given that the child spends a majority of his/her time at home. A stimulating environment ensures that learning is reinforced in a safe environment with trusted adults, which, in turn leads to sustained learning. However, creating ECCE supporting home-environment in impoverished communities is easier said than done. SWIT has been successful in increasing its involvement of the community in ECCE through a variety of innovative methods.

The central approach used was generating-demand for ECCE from within the community which would then build support of SWIT's in-preschool interventions. In 2010, the MCV activity in Mumbai was conducted as a demand generation activity to support the ICDS AWCs, with the following specific objectives:

- To increase awareness amongst community about the importance of sending their children to AWCs

- To provide support and recognition to the AWWs for their role as ECE providers in the community

- To promote the use of GGSS teaching and learning materials in the AWCs and at home. 
The MCV in Mumbai targeted communities catered to by one thousand AWCs. Two episodes of GGSS were shown during the MCV - one on the importance of healthy habits: personal hygiene, hand-washing etc. and the other on pre-literacy skills. The message at the beginning and end of the show emphasized the need for children aged three to five to attend AWCs regularly for a continued 'whole child development'.

Over eighteen-hundred MCV shows were held in and around one thousand and forty seven AWCs. These shows were watched by more than twenty eight thousand children between two and eight years old over a period of three months. In addition, more than eight thousand caregivers also attended the shows. Supporting the MCV activity were community level meetings which were attended by more than two thousand five hundred caregivers (iland informatics, 2010; 2011).

A feedback register was maintained for each of the slum visited. AWWs and community members were encouraged to provide their response/suggestions in this register. During the implementation period (October 2010-January 2011) case studies and feedback was collected by the implementation agency.

Over-all the response was encouraging. Some felt reassured that their children will now get quality education through the AWCs, and others promised to become more actively involved in with their local AWCs.

By the end of three months:

1. Community members could now relate to ECCE through AWCs;

2. AWWs felt more empowered within the community to make a difference in the lives of these children;

3. Children were exposed to joyful-learning experiences, critical for their development and therefore more willing to attend pre-school.

Interestingly, feedback from the MCV and interest expressed by the AWWs and caregivers for more information and tips on activities which were appropriate for early childhood development gave impetus to the design and inclusion of special materials for caregivers as well. A good quality ECCE program goes beyond the centre based intervention and involves parents \& community members (stakeholders) responsible for child's learning at home, early stimulus of the child is very important and caregiver material provides caregivers with tools and ideas to engage with their children at home.

Two kits are being provided to over five thousand AWCs in Mumbai this year via; the Shapes Kit focuses on building pre-numeracy concepts and the Nutrition Kit supplements the critical nutritional service provided by the AWCs to mothers as well as children. Each kit contains thirty sets of materials for parents. Given that most caregivers are either illiterate or have low literacy levels special materials have been designed that are easy to understand and use.

Outcomes of the SWIT intervention include AWWs who spend at least half a day counseling the mothers on better health, hygiene and nutrition practices having access to an additional resource for sharing information. The activities help the caregiver undertake specific tasks with their children at home. This not only increases knowledge of the caregiver on the kind of information a child in pre-school age must learn, but also enhances their participation in the child's development.

Taking this intervention further is the introduction of a telephone based support system for the caregivers.

Already established for the AWW, the telephone based system works as follows:

1. Caregivers are registered to receive automated calls from Galli Galli Sim Sim

2. Twice every month, caregivers receive calls from Galli Galli Sim Sim with tips on specific activities which can be carried out with the children. The system allows for the listener to hear the activity as many times as required, 
without having to bear call charges.

3. Caregivers also have the option of leaving messages, suggestions or their success stories on the material or on their learning through the AWCs

4. Through intensive on-ground support, caregivers are encouraged to carry out activities with their children and leave messages about their experiences, which can then be broadcast back to the audience for community-success story inspiration.

The telephone based support for the caregivers is being piloted in a sub-sample of the program area. These materials/telephone based activities are designed to provide the parents/caregivers with an appropriate vocabulary (including use of encouragement and praise) while conducting activities with children.

\section{AWW Capacity Building}

An AWW plays a very crucial role in the growth and development of a child especially from infancy to when the child is ready to begin primary school. It is the AWW, who provides information to a pregnant mother about the need for sufficient nutrition and safe delivery through home visits and counseling sessions. The AWW ensures that a new born baby is registered at an AWC and receives routine immunization. The AWW also ensures that the mother continues to receive supplementary nutrition. It is, therefore, imperative to recognize the importance of pre-school services provided by an AWW.

The pre-school component provided by the ICDS also aids with preparation of children for primary schooling and offers substitute care options to younger siblings (historically saddled with the responsibility of taking care of the youngest child) thus, freeing the older ones - especially girls to attend school. The AWW is expected to take-on and execute a wide-variety of ECCE tasks including organization of preschool education activities for three hours a day, attending to peripheral activities such as the preparation of classroom materials, establishing functional links with primary school teachers and maintaining classroom attendance records.

Charts, blocks, picture books, toys and counting frames (SWIT, 2011) are the most common TLM resources available to an AWW. AWWs, at the beginning of their tenure undergo training on use and further development of materials for children (SWIT 2011). They are also required to undergo refresher trainings every two years. This policy of refresher training when coupled with the large number of AWWs creates an almost unending back-log of freshers, given that the rate of re-training is never quite the same as the rate of pedagogical innovation. As a result, most AWWs continue to use outdated materials and methods with no or little access to new ideas, innovations which improve a child's experience in pre-school. Understanding that AWWs are not only over-burdened but also have had very limited exposure to new and innovative learning materials, SWIT has put in place a rigorous capacity development model for these five thousand one hundred and twenty eight AWWs \& their supervisors. The model is based on the premise; if AWWs have access to resources, which guides them, works as a sounding board for ideas, it will motivate them to use existing materials and their learning with the children in innovative ways. This, in turn, translates into joyful learning experiences for the child.

In order to provide continued supportive supervision, SWIT has put in place the following 'cascaded-model'16 of capacity development:

- LEVEL 1 - SWIT has identified and trained a group of people from within the community who have taken on the role of Training Specialist (TS).

16 A type of social intervention design in which a) information/training is in layers or passes on from a small group to a larger group through participatory means and b) impact cascades downwards e.g. trainer to teacher to child to caregiver. 
A group of fifteen young women from Mumbai, this year, underwent a rigorous four-day training which covered concepts of ECCE, the role and importance of joyful and child friendly materials ways to improve ECCE messaging between the AWW and the child. In a sub-set of the program area, the training specialists work closely with the AWW to build their capacities as ECCE providers in the community.

- LEVEL 2 - Each AWW reports to a Supervisor. In ICDS Mumbai, there are over one hundred and sixty supervisors who each manage between thirty-thirty two AWWs. Since the supervisors not only meet the AWW on a regular basis (through field visits, meetings, reporting, and etc.), but provide on the job training, they became an obvious choice for master trainers. A hundred supervisors were therefore shortlisted and trained as GGSS master trainers over a period of two days. These trainings were conducted by a team of four training specialists for every twenty five master trainers. This training model ensured the following:

1) Each master trainer has a one-point contact for any queries regarding kits, training or feedback from the AWW

2) Each training specialist is responsible for ensuring that trainings are held on time, as per a predefined schedule and in an appropriate fashion

3) Each training specialist is able to attend trainings conducted by master trainers, observe \& provide instantaneous feedback

- LEVEL 3 - Master trainers were then required to train five thousand one hundred and twenty eight AWWs. The training schedule is scheduled such that each master trainer conducts two-three trainings for a batch of twenty-five to thirty AWWs each. Each batch undergoes training for a day with a focus on improving quality of ECCE delivery with children and interaction with caregivers.

Pre and post training surveys (SWIT, 2011) are also conducted across levels and have shown significant improvement in the understanding ECCE and in recognizing the caregiver's role in a child's over-all development. SWIT believes that a high-touch, highimpact, capacity-building model such as the above will definitely improve the quality of interaction with children in the center resulting in improved access to joyful learning experiences for every child during their pre-school years.

\section{Joyful Learning Experiences}

The National Curriculum Framework (NCERT, 2005) document clearly establishes the need for an activity based curriculum according to the needs, interests and age of children. The curriculum framework discourages the use of formal teaching and highlights the importance of play as the basis of learning.

To ensure all round development and make learning fun for children aged between three and six year old, SWIT has developed innovative and high quality print materials on different themes. These materials provide tactile stimulation to children (children can touch \& feel) and are designed with colorful attractive pictures to capture children's attention and sustain interest. A range of colorful child held materials like puzzles, cut-outs, activity books, and etc. ensure that they actively learn different concepts through fun and engaging activities.

To build the capacity of AWWs and ensure that they conduct developmentally appropriate activities with young children, SWIT has created a curriculum framework that aligns with their daily AWW schedule. Keeping in mind the developmental trajectory of children, separate curriculum documents are created for 3-4 year olds and 4-6 year olds. These frameworks provide the AWWs with an activity she can conduct with children in her class with (or 
sometimes without) GGSS print materials.

Learning is the change of behavior acquired by individual experiences. Joyful student-centered learning experiences positively influence a child's interest creativity, initiative and confidence, which are critical skills for a successful life.

\section{Conclusions and Recommendations}

In conclusion, SWIT's work in Mumbai demonstrates that a combination of quality teacher training, bottom-up programme design, inclusion of community members as drivers of change and advocacy with caregivers - significantly contribute to improving learning levels in children between the ages of birth and six. Designing and operating such a programme at scale requires a smaller scale pilot which allows for mid-course correction, content which is based on formative research conducted incommunity settings and a rigorous ME\&R process to understand its impact.

\section{References}

AC Nielsen \& Company. (2011). Television Audience Measurement (TAM) media research. Mumbai: Author.

BGM Policy Innovations. (2010, November). Understanding our civic issues- early childhood care and education. Paper presented at an evaluation of project metro for Sesame Workshop India. Mumbai, India.

ICDS Maharashtra. (2009). About ICDS (Integrated Child Development Services Scheme). Retrieved from http://www.icds.gov.in/\#

iland informatics. (2010, November). An evaluation of mobile community viewings. Paper presented at an evaluation of project metro for Sesame Workshop India. Mumbai, India.

Kaul, V., Sankar, D. (2009). Education for all- mid decade assessment: Early childhood care and education in India. New Delhi, India: National University of Educational Planning and Administration.

United Nations. (2009). The millennium development goals report 2009. New York: United Nations.

National Council of Educational Research and Training [NCERT]. (2005). National curriculum framework document. New Delhi, India: Author.

NCERT. (2006). National focus group on early childhood education (Position Paper No. 36). New Delhi, India: Author.

Gragnolati, M., Shekar, M., Gupta, M. D., Bredenkam p, C., \& Lee, Y. K. (2005). Chapter 2 The Integrated Child Development Services Program (ICDS)-Are results meeting expectations? In India's undernourished children: A call for reform and action. Retrieved from http://siteresources.world bank.org/SOUTHASIAEXT/Resources/22354611 47272668285/undernourished_chapter_2.pdf

Sesame Workshop India Trust. (2011). Internal monitoring reports. New Delhi, India: Author.

UNESCO. (2002). EFA Global Monitoring Report 2002: Education for all: Is the world on track. Paris: Author. Vora, A. (2010, December 13). Mumbai's silent pangs. Hindustan Times. Retrieved from http://www. hindustantimes.com/India-news/Mumbai/ Mumbai-s-silent-pangs/Article1-637695.aspx

Ministry of Women and Children Development [WCD]. (2011, January). Agenda for conference of state ministers in charge of women \& child development. Paper presented at the Conference of state ministers in charge of women \& child development. New Delhi, India: WCD, Government of India. 